\title{
Retrospective evaluation of the BIG score to predict mortality in pediatric blunt trauma
}

\author{
Charlotte Grandjean-Blanchet, $\mathrm{MD}^{*}$; Guillaume Emeriaud, MD*; Marianne Beaudin, $\mathrm{MD}^{\dagger}$; \\ Jocelyn Gravel, MD, MSc*
}

\section{ABSTRACT}

Objectives: This study's objective was to measure the criterion validity of the BIG score (a new pediatric trauma score composed of the initial base deficit [BD], international normalized ratio [INR], and Glasgow Coma Scale [GCS]) to predict in-hospital mortality among children admitted to the emergency department with blunt trauma requiring an admission to the intensive care unit, knowing that a score $<16$ identifies children with a high probability of survival.

Methods: This was a retrospective cohort study performed in a single tertiary care pediatric hospital between 2008 and 2016. Participants were all children admitted to the emergency department for a blunt trauma requiring intensive care unit admission or who died in the emergency department. The primary analysis was the association between a BIG score $\geq 16$ and in-hospital mortality.

Results: Twenty-eight children died among the 336 who met the inclusion criteria. Two hundred eighty-four children had information on the three components of the BIG score, and they were included in the primary analysis. A BIG score $\geq 16$ demonstrated a sensitivity of 0.93 (95\% confidence interval [Cl]: $0.76-0.98)$ and specificity of $0.83(95 \% \mathrm{Cl}: 0.78-0.87)$ to identify mortality. Using receiver operating characteristic curves, the area under the curve was higher for the BIG score $(0.97 ; 95 \%$ IC: $0.95-0.99)$ in comparison to the Injury Severity Score (0.78; 95\% IC: 0.71-0.85).

Conclusion: In this retrospective cohort, the BIG score was an excellent predictor of survival for children admitted to the emergency department following a blunt trauma.

\section{RÉSUMÉ}

Objectif: L'étude visait à mesurer la validité critérielle du score BIG (nouvelle échelle de gravité des traumas chez les enfants, fondée sur le déficit en base (DB), le rapport international normalisé (RIN) et l'échelle de Glasgow à l'évaluation initiale) quant à sa valeur prévisionnelle de la mortalité hospitalière chez les enfants admis au service des urgences (SU) pour des traumas contondants, puis traités au service des soins intensifs; en effet, un score $<16$ est annonciateur d'une forte probabilité de survie.
Méthode: II s'agit d'une étude de cohorte rétrospective, menée dans un seul centre hospitalier de soins tertiaires pour enfants, entre 2008 et 2016 . Tous les participants étaient des enfants admis au SU pour des traumas contondants et qui ont été traités au service des soins intensifs ou qui sont morts au SU. L'analyse principale consistait en I'association entre un score $\mathrm{BIG} \geq 16$ et la mortalité hospitalière.

Résultats: Trois cent trente-six enfants respectaient les critères de sélection et, sur ce nombre, 28 sont morts. Nous disposions de renseignements sur les trois éléments composant le score BIG chez 284 enfants, et ces derniers ont été retenus dans l'analyse principale. II s'est révélé qu'un score BIG $\geq 16$ avait une sensibilité de 0,93 (IC à $95 \%: 0,76-0,98$ ) et une spécificité de 0,83 (IC à $95 \%: 0,78-0,87$ ) au regard de la mortalité. La valeur de la surface sous la courbe du score BIG $(0,97$; IC à $95 \%$ : 0,95-0,99), d'après les courbes ROC, était plus élevée que celle de l'indice de gravité des blessures ("ISS " en anglais) (0,78; IC à $95 \%: 0,71-0,85)$.

Conclusion: Dans cette étude de cohorte rétrospective, le score BIG a révélé une excellente valeur prévisionnelle quant à la survie des enfants admis au SU pour des traumas contondants.

Keywords: blunt trauma, children, intensive care unit, trauma scores, mortality, pediatrics, emergency medicine

\section{INTRODUCTION}

Traumatic injuries are the leading cause of death among children in Canada and other developed countries. According to the World Health Organization, in 2004, nearly 950,000 children below the age of 18 died across the world as a result of injury. As for Canada, according to the Public Health Agency of Canada, 634 young Canadians ages 19 and under were killed by an injury in $2008 .^{1-4}$

To better characterize the morbidity engendered by trauma, numerous pediatric trauma scoring systems have been developed in the past few years. ${ }^{5-9}$ However, these trauma scoring systems are often complex,

From the *Division of Pediatrics; and tDivision of Surgery, Centre hospitalier universitaire (CHU) Sainte-Justine, Montreal, QC.

Correspondence to: Dr. Charlotte Grandjean-Blanchet, CHU Sainte-Justine, Division of Pediatrics, 3175 Chemin Côte Sainte-Catherine, Montreal, OC H3T 1C5; Email: cgblanchet@hotmail.com 
requiring time and trained personnel. Also, they lack validity when applied to different populations, leading to their inconsistent use in pediatric trauma centres. ${ }^{7}$

The BIG score is composed of the initial base deficit (BD), international normalized ratio (INR), and Glasgow Coma Scale (GCS), three variables that play a critical role in children's survival. It is calculated as follows:

$$
\text { BIG score }=(\mathrm{BD})+(2.5 \times \mathrm{INR})+(15-\mathrm{GCS})
$$

The BIG score has been recently developed among 707 children admitted to combat-support hospitals in Iraq and Afghanistan and validated among 1,101 children admitted to a German hospital. ${ }^{10}$ Following these, the BIG score was validated retrospectively using the trauma database of a single tertiary care pediatric hospital. ${ }^{11}$

The BIG score predicted mortality in children with traumatic injuries, with a high probability of survival in patients with a score of $<16 .{ }^{11}$ This new score is easy to calculate and outperformed previous trauma scoring models in two retrospective studies. ${ }^{10,11}$

To be widely accepted and disseminated, the BIG score will have to prove validity in multiple settings. The aim of this study was to measure the criterion validity of the BIG score to predict in-hospital mortality among children with blunt trauma admitted to a pediatric emergency department (ED).

\section{METHODS}

This was a retrospective cohort study performed between 2008 and 2016 in a single tertiary care pediatric hospital serving a large territory. During the study period, the ED had an annual census varying between 70,000 and 84,000 patient visits per year, with approximately 40 admissions per year at the pediatric intensive care unit (ICU) for traumatic injuries. The study was approved by the research ethics board of our centre.

\section{Study population}

Potential participants were all consecutive children admitted to the ED for a blunt trauma. To improve resource utilization, the study was focused on relatively severe patients, namely trauma patients admitted to the pediatric ICU or those who died in the ED. Participants were identified using the Quebec Trauma Registry Information System (SIRTQ). This computerized trauma database is managed by the Ministry of Health and Social Services and undergoes periodic evaluations to identify incorrect data values. It has been used since 1998 to improve the quality of care of trauma patients in Quebec. Trauma patients included in this registry are patients who either died following trauma, were admitted to the ICU, were hospitalized for more than 2 days, or were transferred from another hospital. Medical archivists use standardized coding protocols to extract the registry data from patients' files. Inclusion criteria were all children less than 18 years of age with a blunt trauma admitted to the ICU or who died at our ED between April 2008 and March 2016. Patients intubated prior to being transferred to the hospital were included if a GCS prior to intubation was available. We excluded children with penetrating trauma as well as patients with known co-morbidities that could influence the BIG score, such as coagulopathies and metabolic, cardiac, and neurologic diseases. Patients who died on the scene or before arriving to the hospital were also excluded.

\section{Outcome}

The primary outcome for this study was the association between in-hospital mortality and an initial BIG score $\geq 16$. Secondary outcomes included the association between the BIG score and the probability of transfer to a rehabilitation centre, and the length of hospitalization.

\section{Independent variables}

Demographic and clinical information included age, sex, mechanism of injury, associated head injury, transfer from a referral hospital, intubation, blood transfusion, need for surgery, length of hospital stay, transfer to a rehabilitation centre, Injury Severity Score (ISS), death, as well as the initial components of the BIG score available at the referral hospital or at our hospital (base deficit, $\mathrm{INR}$, and pre-intubation GCS). The BIG score was calculated as $(\mathrm{BD})+(2.5 \times \mathrm{INR})+(15-\mathrm{GCS}) .{ }^{10,11}$

\section{Data collection}

All charts were reviewed by a member of the research team (CGB), using a standardized report form. This form included information on date of trauma, patient's age, patient's sex, mechanism of injury, associated head injury, transfer from a referral hospital, intubation, 
transfusion, admission to ICU, surgery, length of hospitalization, death, transfer to a rehabilitation centre, and the three components of the BIG score. To ensure the quality of data abstraction, $10 \%$ of the charts were reviewed in duplicate by a second rater (JG) blinded to the first evaluation.

\section{Data analysis}

Analyses were performed with SPSS v21 software (SPSS Inc., Chicago, Illinois). The primary analysis was the association between the BIG score and mortality for all children for whom all three components of the BIG score were available. Sensitivity and specificity were calculated for a BIG score $\geq 16$. Secondary analyses included the comparison of the BIG score with the ISS. To do so, receiver operating characteristic (ROC) curves were constructed to compare the area under the curve (AUC) of the BIG score with the other scores. Other secondary analyses were to calculate the sensitivity and specificity of the individual components of the BIG score and to evaluate the associations between the
BIG score and the probability of transfer to a rehabilitation centre as well as the length of hospitalization using logistic and linear regression analysis. As for children with missing components of the BIG score, an analysis was conducted while imputing missing data by normal values (INR: 1.1, BD: 1.0 ). The $95 \%$ confidence interval (CI) was calculated for each measurement.

\section{Sample size}

It was calculated that the inclusion of at least 25 deaths would provide CIs of $+/-0.20$ for proportions in the worst-case scenario.

\section{RESULTS}

Three hundred ninety-seven patients who suffered from a traumatic injury between April 2008 and March 2016 and who were admitted to the ICU or died in the ED were identified with the computerized trauma database of our hospital. Of these, 336 met the inclusion criteria (Figure 1). Fifty-one (15\%) patients had missing

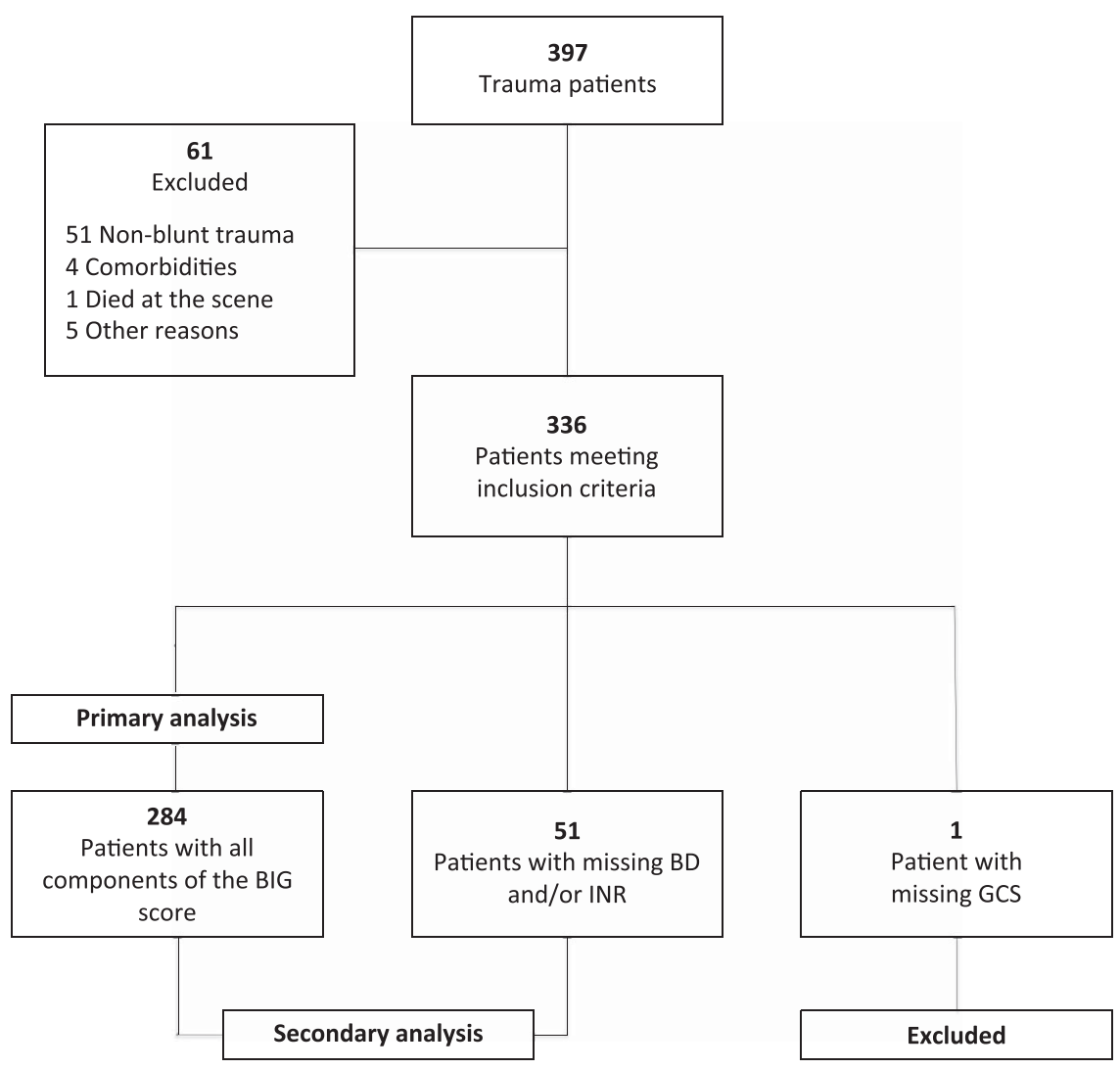

Figure 1. Our study population included all pediatric blunt trauma patients who met the inclusion criteria from our hospital between April 2008 and March 2016. 


\begin{tabular}{|c|c|c|}
\hline & $\begin{array}{c}\text { All patients } \\
n=336\end{array}$ & $\begin{array}{l}\text { Patients without missing data } \\
\text { for the BIG score } \\
n=284\end{array}$ \\
\hline Sex male (\%) & $218(65)$ & $184(65)$ \\
\hline Median age (1st and 3rd quartiles) & $9(5-13)$ & $10(5-13)$ \\
\hline \multicolumn{3}{|l|}{ Mechanism of injury (\%) } \\
\hline - Fall & $73(22)$ & $56(20)$ \\
\hline - Motor vehicle incident & $99(29)$ & $82(29)$ \\
\hline - Physical abuse & $30(9)$ & $28(10)$ \\
\hline - Bicycle/scooter/ATV incident & $41(12)$ & $35(12)$ \\
\hline - Suicide attempt by hanging & $9(3)$ & $9(3)$ \\
\hline - Other & $84(25)$ & $74(26)$ \\
\hline Patient intubated (\%) & $189(56)$ & $180(63)$ \\
\hline Transfer from another facility (\%) & $274(82)$ & $230(81)$ \\
\hline Associated head injury (\%) & $269(80)$ & $231(81)$ \\
\hline Need for surgery (\%) & $130(39)$ & $110(38)$ \\
\hline Blood transfusion (\%) & $102(30)$ & 94 (33) \\
\hline Median Injury Severity Score (1st and 3rd quartiles) & $18(10-26)$ & $19(10-29)$ \\
\hline Transfer to a rehabilitation centre (\%) & $57(17)$ & $53(19)$ \\
\hline Death (\%) & $28(8)$ & $27(10)$ \\
\hline
\end{tabular}

data for INR and/or BD. These patients were excluded from our primary analysis and included subsequently in our secondary analysis. One patient had a missing GCS and was excluded from the primary and secondary analysis because it seemed inappropriate to put a normal value to its GCS according to the medical chart.

The median (interquartile range $[\mathrm{IQR}]$ ) age of the study population was 9 years (IQR), and $65 \%$ were male. As described in Table 1, the most frequent mechanisms of injury were motor vehicle incidents (27\%) followed by falls from height (22\%). The median ISS was 18 (IQR).

The overall mortality rate was $8.3 \%(\mathrm{n}=28)$. One patient died in the ED, and 27 died in the ICU. Of the 28 non-survivors, 12 (43\%) had a non-accidental injury (child abuse and suicide by hanging), 12 (43\%) were involved in a motor vehicle incident, and the remaining suffered from another type of blunt trauma (Table 2).

The inter-rater agreement for data abstraction was excellent for the 36 charts reviewed in duplicate with kappa scores or intraclass correlation coefficients (ICC) $>0.8$ for all data. The inter-rater agreement for a BIG score of $\geq 16$ was perfect.

The BIG score was associated with mortality (Table 3). Of the 284 patients without missing data for the BIG score components, 215 patients had a BIG score of $<16$. Only two $(1 \%)$ of these patients died. A BIG score of $\geq 16$ demonstrated a sensitivity of 0.93
(95\% CI: $0.76-0.98)$ and a specificity of 0.83 (95\% CI: $0.78-0.87)$ to identify mortality.

Sensitivity and specificity to identify mortality were also calculated for the individual components of the BIG score. The three individual components of the BIG score demonstrated sensitivities varying between 0.77 and 0.93 , whereas the specificities varied between 0.66 and 0.74 (Table 4).

ROC curve for predicting mortality using the BIG score is shown in Figure 2, $A$ (continuous BIG score) and Figure 2, $B$ (dichotomized BIG score). The AUC for these ROC was 0.97 (95\% IC: 0.95-0.99) and 0.88 (95\% IC: 0.81-0.94), respectively. The AUC for the ISS (Figure 3) was 0.78 (95\% IC: 0.71-0.85).

The BIG score was associated to other markers of severity. This is demonstrated by the statistical association between the BIG score and length of hospital stay among survivors using linear regression $(p<0.001)$. As a mean, for each increase of 1 point in the BIG score, the duration of hospitalization increased by 0.9 days (95\% CI: 0.5-1.2). Also, using logistic regression, a BIG score of $\geq 16$ was strongly associated to the probability of transfer to a rehabilitation centre for the patients who survived (odds ratio: 6.57 95\% CI: 3.24-13.3).

A secondary analysis imputing all missing data as normal for the 51 patients with missing data for INR and/or BD resulted in a sensitivity of 0.93 
Table 2. Demographic and clinical characteristics of survivors versus non-survivors $(\mathbf{n}=336)$

\begin{tabular}{lcc}
\hline & Survivors & Non-survivors \\
& $\mathrm{n}=308$ & $\mathrm{n}=28$ \\
\hline Sex male (\%) & $201(65)$ & $17(61)$ \\
Median age (1st and 3rd quartiles) & $9(5-13)$ & $11(4.5-14)$ \\
Mechanism of injury (\%) & & 0 \\
- Fall & $73(24)$ & $12(43)$ \\
- Motor vehicle incident & $96(31)$ & $6(21)$ \\
- Physical abuse & $24(8)$ & $3(11)$ \\
- Bicycle/scooter/ATV incident & $28(9)$ & $6(21)$ \\
- Suicide attempt by hanging & $3(1)$ & $1(3)$ \\
- Other & $84(27)$ & $28(100)$ \\
Patient intubated (\%) & $161(53)$ & $26(93)$ \\
Transfer from another facility (\%) & $248(81)$ & $21(75)$ \\
Associated head injury (\%) & $248(81)$ & $5(18)$ \\
Need for surgery (\%) & $125(41)$ & $19(68)$ \\
Blood transfusion (\%) & $83(27)$ & $27(25-45)$ \\
Median Injury Severity Score (1st and 3rd quartiles) & $17(10-26)$ & $27.3(24.3-37.1)$ \\
Median BIG score (1st and 3rd quartiles) & $7.8(4.3-12.5)$ &
\end{tabular}

Table 3. Outcomes for patients with all components of the BIG score $(n=284)$

\begin{tabular}{|c|c|c|c|}
\hline & Survivors & Non-survivors & \\
\hline $\mathrm{BIG} \geq 16$ & 44 & 25 & 69 \\
\hline \multirow[t]{2}{*}{$\mathrm{BIG}<16$} & 213 & 2 & 215 \\
\hline & 257 & 27 & 284 \\
\hline
\end{tabular}

Sensitivity: $0.93 \mathrm{Cl}: 0.77$ to 0.98

Specificity: $0.83 \mathrm{Cl}: 0.78$ to 0.87

$\mathrm{BIG}=$ composed of base deficit, international normalized ratio, and the Glasgow Coma Scale.

\begin{tabular}{|c|c|c|c|}
\hline & Glasgow $<9$ & INR $>1.2$ & Base deficit $>5$ \\
\hline Sensitivity & $0.93(0.77-0.98)$ & $0.77(0.59-0.89)$ & $0.85(0.68-0.94)$ \\
\hline Specificity & $0.73(0.66-0.77)$ & $0.66(0.60-0.71)$ & $0.74(0.69-0.79)$ \\
\hline
\end{tabular}

(95\% CI: $0.77-0.98)$ and a specificity of 0.86 (95\% CI: 0.81-0.89) to identify mortality.

\section{DISCUSSION}

The BIG score predicted in-hospital mortality among children suffering from a blunt trauma with a sensitivity of 0.93 and a specificity of 0.83 in a study population different from where it was derived and previously validated. A BIG score of $<16$ identified children with a high probability of survival. This remained similar when adding the patients for whom a physiological component (BD and/or INR) was missing and imputed as normal.

Our results are consistent with previous findings that validated the BIG score as a mortality predictor in pediatric blunt trauma patients, independently of prehospital intubation, fluid resuscitation, and head injury. ${ }^{11}$

Unlike numerous pediatric trauma scoring systems currently used, the BIG score constitutes three physiological measurements that play a critical role in children's survival. Trauma-associated coagulopathy has been well studied in adults. A quarter of severely traumatized adult patients have abnormal blood coagulation when they arrive in the ED, which is associated with a higher risk of mortality. ${ }^{12,13}$ This early coagulopathy has been referred to as acute traumatic coagulopathy, early coagulation of trauma, trauma-induced coagulopathy, and acute coagulopathy of trauma-shock. ${ }^{14,15}$ This coagulopathy is primarily caused by the endothelial activation of the protein $\mathrm{C}$ pathway influenced by the combination of tissue injury and shock. This leads to rapid anticoagulation and fibrinolysis, which put the trauma patient at risk of severe hemorrhage. ${ }^{15-17}$ Other factors influencing coagulation have also been described, such as hemodilution of coagulation factors, which is caused by crystalloid administration during resuscitation, hypothermia, acidosis, 

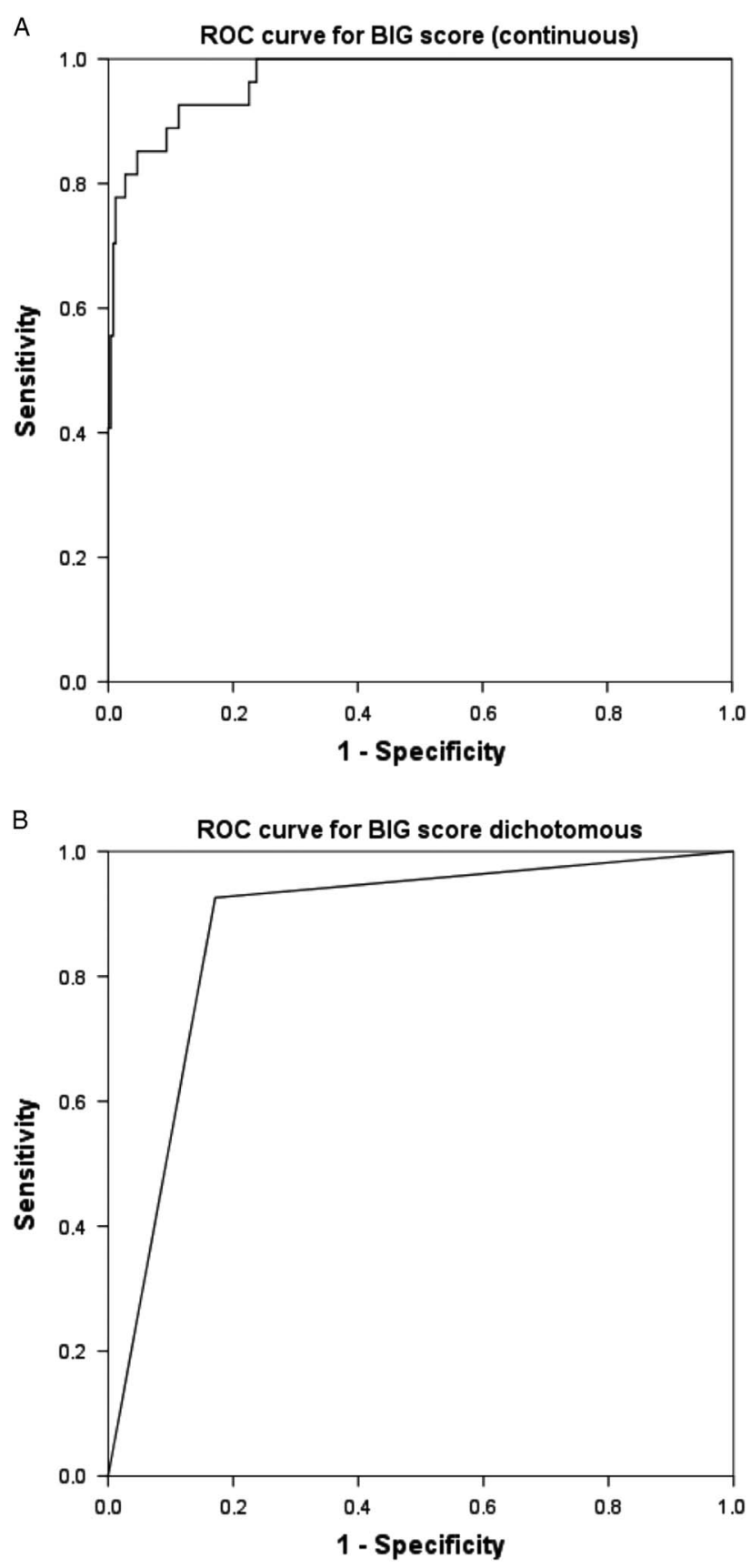

Figure 2. A) ROC curve for the BIG score (continuous) versus mortality. B) ROC curve for the BIG score (higher or lower than 16) versus mortality.

anemia, and hypocalcemia. ${ }^{13,15,18}$ Thus, tissue injury and shock are the main drivers of early coagulopathy, whereas other factors exacerbate the coagulopathy. ${ }^{15,18}$ Hemodilution, hypothermia, and acidosis as contributing factors to coagulopathy have also been described in the pediatric population. However, the mechanism explaining early coagulopathy in traumatized pediatric patients remains unknown. According to Whittaker

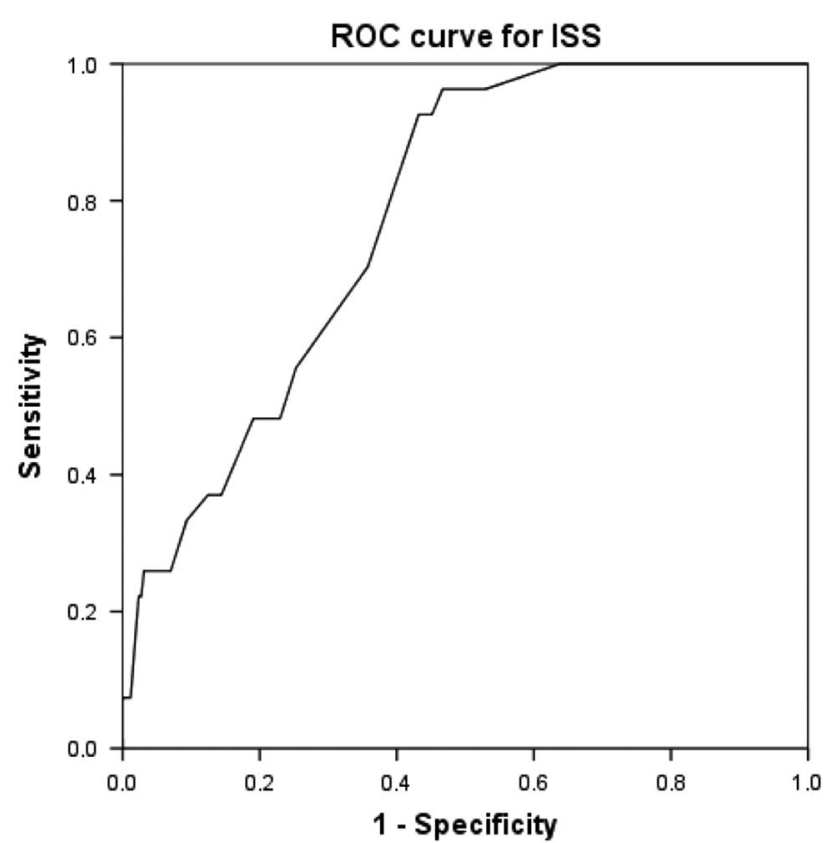

Figure 3. ROC curve for the ISS versus mortality.

et al., early coagulopathy is an independent predictor of mortality in severe civilian pediatric trauma patients, particularly in patients with traumatic brain injury (TBI). ${ }^{13}$ TBI-associated coagulopathy has been described in a few studies, and several hypotheses have been proposed to explain this association but remain unproven to this day. ${ }^{19-21}$ Strumwasser et al. also found that coagulopathy was associated with an increased risk of death in pediatric patients. According to their study, trauma coagulopathy in pediatric trauma patients tends to develop later compared to adults, suggesting that early coagulopathy in children may be associated with increased mortality. ${ }^{22}$

The GCS is used across the world as a tool to assess patients' level of consciousness, reflecting their degree of brain injury or cerebral hypoperfusion. ${ }^{23}$ TBI being the most common cause of death in trauma patients, GCS is of great interest in predicting mortality in pediatric trauma patients. ${ }^{10,24}$ Cicero et al. reported GCS as an independent predictor of mortality in pediatric trauma victims. ${ }^{23} \mathrm{~A}$ recent study by Yousefzadeh-Chabok et al. compared the GCS to the pediatric trauma score (PTS) and ISS and found that it was a better predictor of mortality in pediatric trauma patients. ${ }^{25}$

As for the base deficit, it reflects the shock status in pediatric trauma patients and has been proven to correlate with injury severity, morbidity, and mortality. ${ }^{26-29}$

The BIG score can be rapidly available on admission to the $\mathrm{ED}$, and it does not require trained personnel to 
be calculated. With an AUC of 0.97 in our study, the BIG score outperformed the ISS $(\mathrm{AUC}=0.78)$. The BIG score performance to predict mortality was significantly better than the INR and BD alone. As for the GCS alone, the sensitivity was the same, but the specificity was lower for the GCS (specificity $=0.73$ ) in comparison to the BIG score (specificity $=0.83$ ). Furthermore, a GCS score of $<9$ with a normal INR and normal $\mathrm{BD}$ was associated with a lower mortality rate (one deceased patient), suggesting that increased physiological disturbance is associated with mortality.

Head trauma was the leading cause of death in our study. However, the BIG score accurately predicted mortality regardless of the cause of death. Furthermore, the BIG score predicted mortality in different populations (U.S. military in Iraq and Afghanistan, Germans as well as Canadians), suggesting that this score can be applied to different blunt traumas as well as to different populations. ${ }^{10,11}$ In practice, the BIG score could help the physicians better assess the patient's physiological state and the severity of its injuries. However, as mentioned by Davis et al., it should not be used to dictate individual treatment. ${ }^{11}$ Regardless of the BIG score, our entire study cohort, except for the patient who died in the ED, was admitted to the ICU, and patients probably could have died without appropriate management. Therefore, the BIG score is more of an additional tool that could give more information about the patient's possible outcome, knowing that an elevated BIG score is associated with increased mortality, a longer hospital stay as well as a higher probability of transfer to a rehabilitation centre. The score would also be useful to stratify patients in clinical trials or to compare case severity for studies using an observational design. Finally, the BIG score could potentially be useful to improve resource allocation.

This study has limitations. Firstly, it is a single site retrospective evaluation of the BIG score, making its external validation less accurate. To be fully validated, the score will need to be evaluated in a multisite prospective study. Several patients included in our study had missing components of the BIG score. To avoid selection bias, patients with missing BD and/or INR were included in a secondary analysis (one patient with a missing GCS was excluded). Of these patients, one died. Despite a missing INR, the BIG score for the non-survivor patient was $>16$. The median ISS for this subgroup was 16 , which is less severe than the median for the subgroup without missing data. Thus, we hypothesized that these patients were not tested for coagulopathy and/or acidosis because their condition was not severe enough, and that, if these tests had been done, they would have been normal. We therefore imputed all missing data as normal for the 51 patients with missing INR and/or BD and included them in a secondary analysis. The sensitivity and specificity of the BIG score to predict mortality were similar for the 284 patients without missing data and all of the patients $(\mathrm{n}=335)$. Furthermore, to minimize missing data, we selected blunt traumas that were admitted to the ICU and that were consequently more likely to have the three components of the BIG score. Thus, our study population was sicker than the general blunt trauma population, leading to potential bias in our test characteristics. However, while artificially improving the specificity of the score by excluding less sick patients, our study design led to the recruitment of all children having the primary outcome (death). Accordingly, the reported sensitivity should be robust. Moreover, the period of time between the trauma injury and the blood tests varied between patients. Blood was drawn to the referral hospital or to our hospital, making it hard to know whether there is an optimal time after trauma to do the blood test. Another limitation is related to the small number of patients with the primary outcome (death) even though we included all children admitted to a very large pediatric ED for 9 years. This limited our ability to conduct multiple analyses. However, the association between the BIG score and other markers of severity suggests that it is a good marker of severity.

\section{CONCLUSION}

The BIG score is a pediatric trauma score that predicts in-hospital mortality in children admitted to the ED following a blunt trauma. A score of $<16$ accurately identifies children with a high probability of survival. The BIG score is a simple tool that can be rapidly available and provides important information about the patient's physiological disturbance and the severity of its injuries. To be widely accepted, the BIG score will need to be validated in a prospective cohort study. However, considering the low mortality rate of children who are victims of blunt trauma, this study will have to involve multiple settings.

Competing interests: None declared. 


\section{REFERENCES}

1. Public Health Agency of Canada. Facts on injury; 2016. Available at: http://www.phac-aspc.gc.ca/injury-bles/factseng.php (accessed 2016).

2. Public Health Agency of Canada. Leading causes of death and hospitalization in Canada; 2016. Available at: http://www.phac-aspc.gc.ca/publicat/lcd-pcd97/index-eng.php (accessed 2016).

3. Peden M. OK, Ozanne-Smith J, Hyder AA, et al. World report on child injury prevention. Geneva: World Health Organization; 2008.

4. Yanchar NL, Warda LJ, Fuselli P. Child and youth injury prevention: a public health approach. Paediatr Child Health 2012;17(9):511-2.

5. Grisoni E, Stallion A, Nance ML, et al. The New Injury Severity Score and the evaluation of pediatric trauma. 7 Trauma 2001;50(6):1106-10.

6. Marcin JP, Pollack MM. Triage scoring systems, severity of illness measures, and mortality prediction models in pediatric trauma. Crit Care Med 2002;30(Suppl 11):S457-67.

7. Ott R, Kramer R, Martus P, et al. Prognostic value of trauma scores in pediatric patients with multiple injuries. 7 Trauma 2000;49(4):729-36.

8. Potoka DA, Schall LC, Ford HR. Development of a novel age-specific pediatric trauma score. 7 Pediatr Surg 2001; 36(1):106-12.

9. Sullivan T, Haider A, DiRusso SM, et al. Prediction of mortality in pediatric trauma patients: new injury severity score outperforms injury severity score in the severely injured. 7 Trauma 2003;55(6):1083-7; discussion 7-8.

10. Borgman MA, Maegele M, Wade CE, et al. Pediatric trauma BIG score: predicting mortality in children after military and civilian trauma. Pediatrics 2011;127(4):e892-7.

11. Davis AL, Wales PW, Malik T, et al. The BIG score and prediction of mortality in pediatric blunt trauma. 7 Pediatr 2015;167(3):593-8e1.

12. MacLeod JB, Lynn M, McKenney MG, et al. Early coagulopathy predicts mortality in trauma. 7 Trauma 2003; 55(1):39-44.

13. Whittaker B, Christiaans SC, Altice JL, et al. Early coagulopathy is an independent predictor of mortality in children after severe trauma. Shock 2013;39(5):421-6.

14. Davenport R. Pathogenesis of acute traumatic coagulopathy. Transfusion 2013;53(Suppl 1):23S-27SS.

15. Hess JR, Brohi K, Dutton RP, et al. The coagulopathy of trauma: a review of mechanisms. 7 Trauma 2008;65(4): 748-54.
16. Christiaans SC, Duhachek-Stapelman AL, Russell RT, et al. Coagulopathy after severe pediatric trauma. Shock 2014; 41(6):476-90.

17. Cohen MJ, Call M, Nelson M, et al. Critical role of activated protein $\mathrm{C}$ in early coagulopathy and later organ failure, infection and death in trauma patients. Ann Surg 2012;255(2):379-85.

18. Fries D, Innerhofer P, Schobersberger W. Time for changing coagulation management in trauma-related massive bleeding. Curr Opin Anaesthesiol 2009;22(2):267-74.

19. Keller MS, Fendya DG, Weber TR. Glasgow Coma Scale predicts coagulopathy in pediatric trauma patients. Semin Pediatr Surg 2001;10(1):12-6.

20. Laroche M, Kutcher ME, Huang MC, et al. Coagulopathy after traumatic brain injury. Neurosurgery 2012;70(6): 1334-45.

21. Talving $\mathrm{P}$, Lustenberger $\mathrm{T}$, Lam L, et al. Coagulopathy after isolated severe traumatic brain injury in children. 7 Trauma 2011;71(5):1205-10.

22. Strumwasser A, Speer AL, Inaba K, et al. The impact of acute coagulopathy on mortality in pediatric trauma patients. 7 Trauma Acute Care Surg 2016;81(2):312-8.

23. Cicero MX, Cross KP. Predictive value of initial Glasgow coma scale score in pediatric trauma patients. Pediatr Emerg Care 2013;29(1):43-8.

24. Dutton RP, Stansbury LG, Leone S, et al. Trauma mortality in mature trauma systems: are we doing better? An analysis of trauma mortality patterns, 1997-2008. I Trauma 2010; 69(3):620-6.

25. Yousefzadeh-Chabok S, Kazemnejad-Leili E, KouchakinejadEramsadati L, et al. Comparing Pediatric Trauma, Glasgow Coma Scale and Injury Severity scores for mortality prediction in traumatic children. Ulus Travma Acil Cerrabi Derg 2016; 22(4):328-32.

26. Jung J, Eo E, Ahn K, et al. Initial base deficit as predictors for mortality and transfusion requirement in the severe pediatric trauma except brain injury. Pediatr Emerg Care 2009;25(9):579-81.

27. Kincaid EH, Chang MC, Letton RW, et al. Admission base deficit in pediatric trauma: a study using the National Trauma Data Bank. 7 Trauma 2001;51(2):332-5.

28. Randolph LC, Takacs M, Davis KA. Resuscitation in the pediatric trauma population: admission base deficit remains an important prognostic indicator. 7 Trauma 2002; 53(5):838-42.

29. Hindy-Francois C, Meyer P, Blanot S, et al. Admission base deficit as a long-term prognostic factor in severe pediatric trauma patients. 7 Trauma 2009;67(6):1272-7. 